\title{
Evaluating progesterone as a novel neuroprotective drug after ocular injury
}

\section{UNIVERSITYOF BIRMINGHAM}

\section{Introduction}

Blunt ocular trauma causes commotio retinae, a retinal opacification that accounts for over a third of all retinal injuries. Ocular trauma is an important preventable public health issue worldwide; injury involving the macula is the most common cause of posttraumatic visual loss and severity is directly proportional to morbidity and socioeconomic demise. ${ }^{1}$

Poor outcomes occur when the macula is affected, which accounts for $1 / 3$ of cases and $3 / 4$ cases in those at high injury risk, causing permanent visual loss due to selective photoreceptor death. This is seen in humans, and reproduced in our animal model, as loss of the outer nuclear layer (ONL) on histological and optical coherence tomography (OCT) images (Figure 1$){ }^{2}$ Loss of outer nuclear

$$
\text { layer }
$$

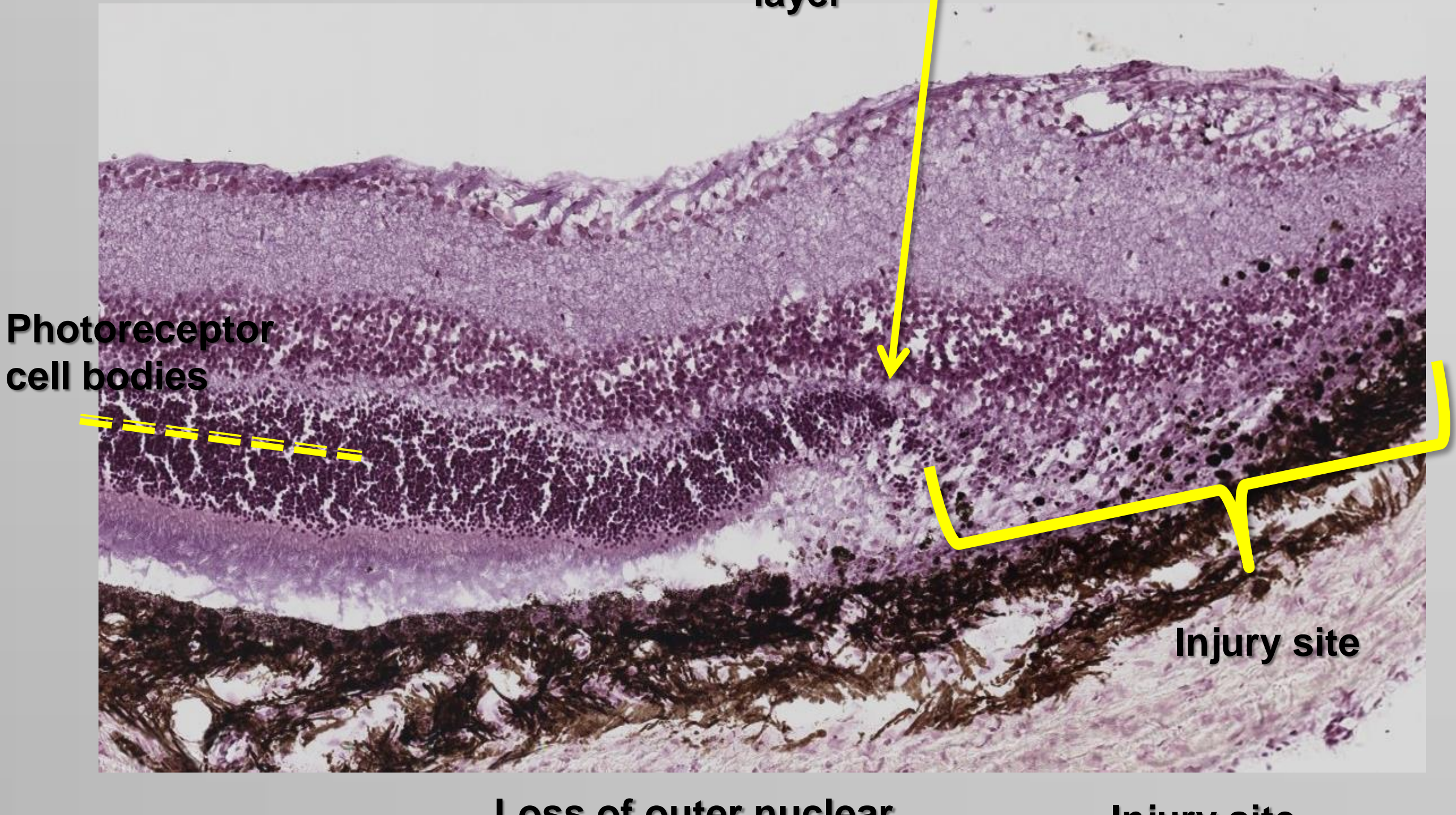

Loss of outer nuclear

Injury site

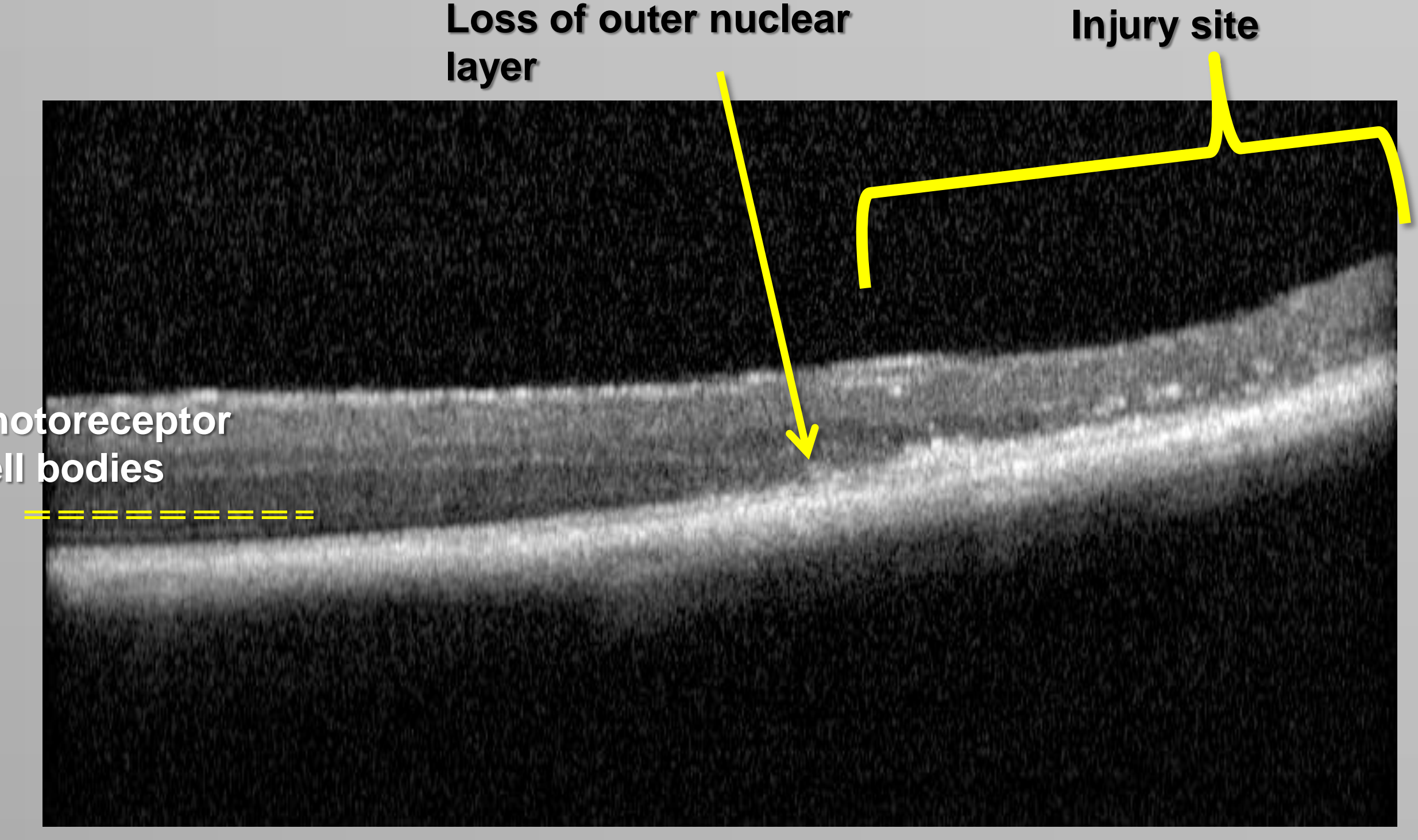

Photoreceptor apoptosis may be prevented and visual outcomes improved by manipulation of cell death pathways using neuroprotective agents. Progesterone has wellestablished neuroprotective effects in various models of CNS injury and there is growing evidence that its pleiotropic actions that interfere with cell death pathways may be extended to ocular trauma. ${ }^{3}$

Figure 1: Haematoxylin stained image (upper panel). OCT image (lower panel). Both rat retinal images taken from this study mark the characteristic features of experimental commotio retinae; specific photoreceptor death at or surrounding the injury site which is seen as loss of the ONL.2

\section{Aim}

The present study aimed to assess the neuroprotective efficacy of progesterone for photoreceptor damage in our experimental model of commotio retinae with respect to histological and functional outcomes.

\section{Methods}

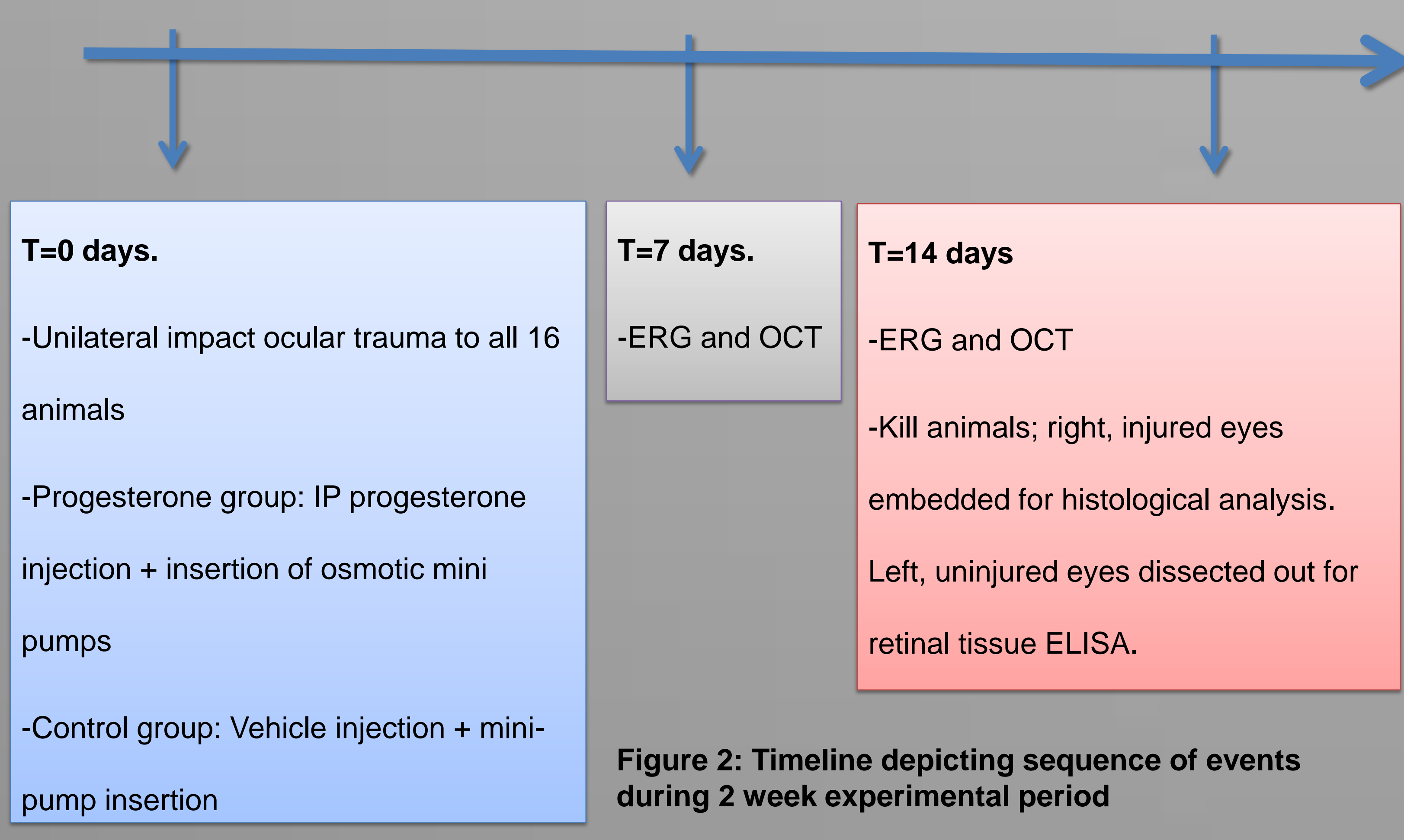

Subsequent to unilateral ocular trauma, half of the animals received progesterone treatment via continuous infusion over the 2-week experimental period.

Electroretinography (ERG) measurements and OCT images were obtained from all animals at 7 and 14 days and ONL thickness was measured from cryoprotected retinal sections at 14 days to assess the degree of photoreceptor death. ONL thickness and awave amplitude were analysed using generalised estimating equations (SPSS v21, IBM, Armonk, NY USA).

\section{Results}

- Progesterone induced significantly greater photoreceptor death $(P=0.002)$, with a more pronounced negative effect apparent at increasing distances from the impact site.

- ERG findings show no overall effect of progesterone treatment. However, there is initial enhancement of photoreceptor function after 7 days, but a negative effect at 14 days $(P=0.001)$.

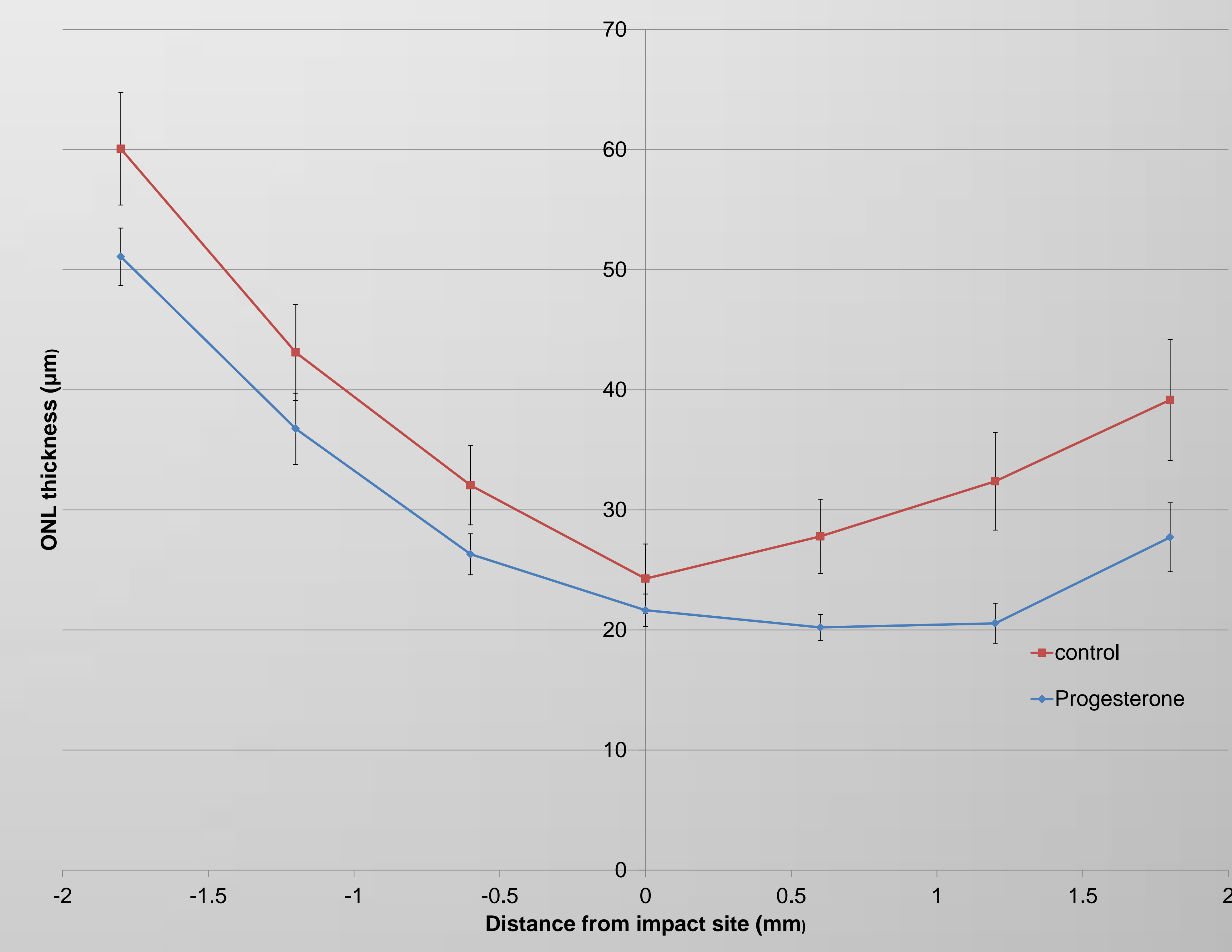

Figure 3: Effect of progesterone treatment on outcomes after ballistic ocular trauma in rat assessed by ONL thickness 14 days after injury. Progesterone treatment decreased $\mathrm{ONL}$ thickness comp
with an effect that increased with increasing distance from the impact site $(p=0.051)$.

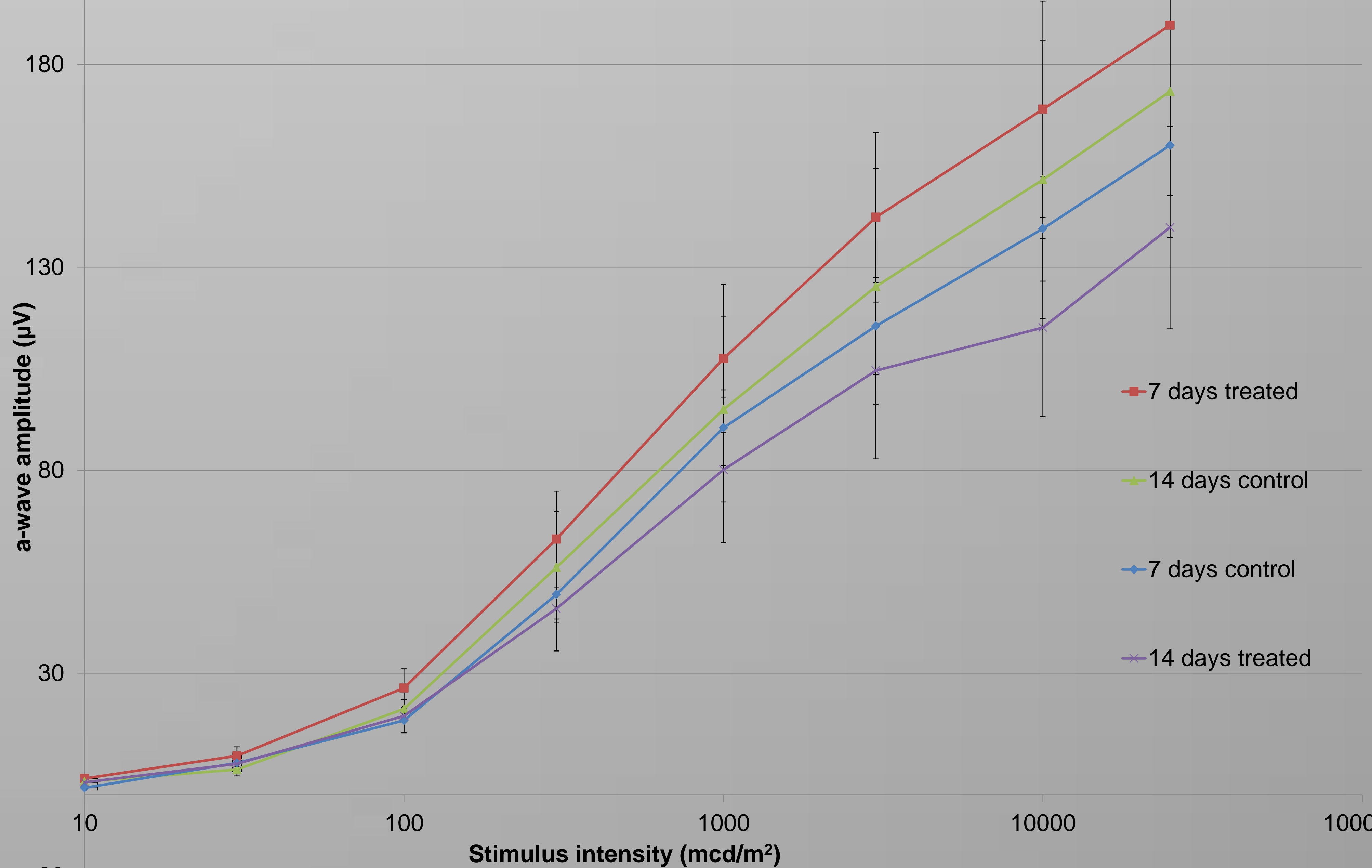

Figure 4: The relationship between increasing stimulus intensity and scotopic 'a' wave amplitude. Treatment with progesterone decreased a-wave amplitude at higher stimulus intensities more than at lower intensities $(p<0.001)$. Treatment with progesterone had a different effect at 7 compared to 14 days $(p=0.001)$, increasing a-wave amplitude compared to controls at 7 days, whilst decreasing it at 14 days. Similar effects were observed in the

\section{Discussion}

With overall increased photoreceptor death, initial enhancement, then deterioration in photoreceptor function, this study is the first to show an apparent neuroprotective effect after short term experimental end points, with progesterone initially delaying apoptosis. This neuroprotective potential demonstrated in others studies may be misleading since at longer-term end points, progesterone treatment had an overall detrimental effect, enhancing apoptosis, highlighting the importance of including longer scale end points in any preclinical study of neuroprotection.

Progesterone increased photoreceptor degeneration after commotio retinae. Whether this was a concentration, or duration-dependent effect requires further investigation.

The increasing effect of progesterone treatment with increasing distance from the impact site suggests that the detrimental effect of progesterone is most pronounced on apoptotic cells, as near to the impact site cells die by necrosis.

\section{References}

1. Kuhn F, Maisiak R, Mann L, et al. The Ocular Trauma Score (OTS). Ophthalmology Clinics of North America 2002;15(2):163-5.

2. Blanch RJ, Ahmed Z, Sik A, et al. Neuroretinal Cell Death in a Murine Model of Closed Globe Injury: Pathological and Functional Characterization. Investigative Ophthalmology \& Visual Science 2012;53(11):7220-7226.

3. Swiatek-De Lange M, Stampfl A, Hauck SM, et al. Membrane-initiated effects of progesterone on calcium dependent signaling and activation of VEGF gene expression in retinal glial cells. Glia 2007;55(10):1061-1073. 